\title{
Fixed dose combinations in indian medicine market - a SWOT analysis
}

\begin{abstract}
Modern medicines are powerful tools to fight against diseases and ailments. Practicing health care professionals like doctors, pharmacists and nurses have the responsibility of promoting and contributing to the rationalization of drug therapy and medicine usage. Promotion of the concept of 'right drug to the right patient at right time in right dose through right route' is important in rational drug therapy. Ideally modern medicines have to be administered as individual items. However situations may arise compelling to combine certain drugs for some specific benefits. Fixed dose combinations (FDC) contain more than one drug in a formulation in a fixed ratio. FDCs are well chosen in certain situations. The introduction of large number of irrational FDCs into the Indian market, without the approval of the national licensing authority has caused to attract strong criticisms from many corners. The issue of irrational FDCs in Indian market is an important topic for critical and scientific analysis. A Committee was constituted by the Government of India in 2014 to study the issue of FDCs, both new and existing and based on its recommendations banned 344 FDC in March 2016. Delhi High Court in December 2016 put a stay on the ban citing technical reasons. In January 2017, Government of India took it to the Supreme Court of India through an appeal against the Delhi High Court verdict. The issue is in the active consideration of Supreme Court. The country is in need of some perpetual mechanism to regulate FDCs after studying whether they are rational and genuinely necessary for our health care. Doctors, Pharmacists and other health care professionals at grass root levels have to be made aware of the benefits as well as ill effects of FDCs. Currently there is no mechanism in the country to provide scientific and authoritative information regarding the FDCs approved by the drugs control general of India. The new drug policy as proposed by the Government of India suggest certain effective measures to curb the menace of FDCs for the first time in the country.
\end{abstract}

Keywords: combination medicines, fixed dose combinations, rational use of medicines, FDCs in india, practicing doctors, practicing pharmacists, irrational combinations
Volume 4 Issue 3 - 2017

\author{
Revikumar KG,' Veena $\mathrm{R}^{2}$ \\ 'Nirmala College of Pharmacy, India \\ ${ }^{2}$ College of Pharmaceutical Sciences, MG University, India
}

Correspondence: Revikumar KG, Principal, Nirmala College of Pharmacy, Kunnappilly, Meloor, Chalakudy, Thrissur, Kerala, 680 3II, India, Email kg.revikumar@gmail.com

Received: December 10,2017 | Published: December 20, 2017
Abbreviations: FDC, fixed dose combinations; ACT, artemisinin-based combination therapy; WHO, world health organization; API, active pharmaceutical ingredient; CDSCO, central drugs standard control organization; SLAs, state licensing authoritie; DCGI, drugs control general of india; DTAB, drugs technical advisory board

\section{Introduction}

Medicines are weapons used to fight ailments and diseases. More than one medicine is often used for treatment of either single ailment or multiple co-morbid conditions. Most of the modern medicines are potent chemicals having specific and characteristic physical, chemical, pharmacological and therapeutic properties. These properties vary from drug to drug. The dose, route and the regimen of a drug vary from one to another based on its intrinsic properties and characteristics. The chemistry of most of the generic molecules are supporting individual use of medicines, rather than their combinations. Practising pharmacists, doctors and nurses constitute the right category of health professionals who can appreciate and apprehend the facts related to modern medicines in the proper perspective and take them to the benefit of patients, health care professionals and the health care programs.

\section{What are combination drugs?}

Ideally drugs have to be administered as individual items based on the specific requirement of each patient. It enables the prescriber to select specific drugs in specific doses for specific durations to individual patients in certain specific conditions. However on certain special occasions or situations we may have to unite together certain drugs for better results, convenience and certain other benefits. In the past such practices were either incident based or individual focused and managed through the compounding process by the pharmacists. When the compounding aspect was taken up by the pharmaceutical firms, the concept of combination of drugs also got a commercial outlook. When two or more drugs are combined together in a fixed ratio into a single dosage from, it is known as Fixed Dose Combinations or FDCs.

\section{Useful FDCs}

In the case of malaria, drugs like chloroquine or sulphadoxinepyrimethamine have become increasingly ineffective due to drug resistance. Scientists and doctors agree that the most effective treatment against malaria is a combination of drugs using artemisinin derivatives, highly potent extracts of the Chinese plant Artemesia annua. This Artemisinin-based combination therapy (ACT) is the 
quickest and most reliable way of clearing malaria infection, and is very well tolerated. ${ }^{1}$ Here use of a combination of drugs shortens the treatment course. WHO has recommended to phase out monotherapy in the treatment of malaria. In certain cases FDCs help to improve the quality of life and also the efficacy of the medicine. They also help to reduce the number of pills taken by the patient at a time. The WHO list of essential drugs contain certain FDCs. Rational combinations are surely of much use to the health care program. Unfortunately accepted uniform norms or standards for the development of FDCs and their potential benefits or disadvantages in treating the diseases are currently lacking. The development of fixed-dose combinations (FDCs) is becoming increasingly important from the public health perspective. FDCs available for the treatment of various ailments range from nutritional deficiency to cardiovascular diseases. In certain cases FDCs help to simplify treatment regimens, improve patient adherence and facilitate implementation of interventional programs and prevent the development of drug resistance. There shall be cases where individual drugs have to be used for certain patients because of the challenges of dose titration, adverse reactions to one or more of the components or different pharmacokinetic and /or pharmacodynamic profiles.

\section{Box no 1 when FDCs are chosen:}

a. Where two or more drugs have a synergistic action. It means that the combination acts to have a better therapeutic response than the individual drugs if used separately. Here the combination will have better pharmacokinetic and pharmacodynamic properties.

b. Where there is a corrective action. Here one drug may act to reduce the incidence, intensity or severity of adverse effects caused by the other.

c. Where two or more drugs are needed to be effective for the proper management of a particular condition. The combination helps for enhancing therapeutic efficacy.

d. Where prescribing two or more drugs separately could result in one of them not being taken or administered, and that could adversely affect the patient's health. The combination will enhance the compliance and adherence.

e. Where the combination can reduce the dose of individual drug or drugs and decrease the development of drug resistance.

f. Where the combination drug is cheaper than individual drugs because of reduced cost from packaging to distribution.

\section{Why FDCs became an issue?}

In a study conducted on 100 resident doctors, it was found that $81 \%$ lacked knowledge about the rationality of given FDCs and only $43 \%$ were able to mention a single banned FDC in India. ${ }^{2}$ A study from Mumbai highlighted the nexus between pharmaceutical companies, medical representatives, chemists and doctors to make profits at the expense of consumers and the public's health. ${ }^{3}$ It is an accepted fact that an FDC be treated as a new drug, because by combining two or more drugs, the safety, efficacy, and bioavailability of the individual Active Pharmaceutical Ingredient (API) can change. According to Rule 122(E) of the Drugs and Cosmetics Act, new combinations are 'new drugs'. During the last few decades, Indian Pharmaceutical companies started to introduce newer and newer FDCs into the market at regular intervals, often without giving due weight age to the professional and scientific aspects of their acceptance. It has caused to create confusion and suspicion among professionals. It is quite natural the issue of FDCs have developed to be a topic of debate and discussion at various levels throughout the world for quite some time. A number of papers and articles were published on the topic, majority of them highlighting the health hazards, unethical aspects and other unhealthy aspects of FDCs. ${ }^{4}$ Still to the surprise of one and all, the numbers of FDCs are increasing in the market year by year both in number and volume.

\section{Box no 2. problems of FDCs formulated with the object of commercial interest:}

a. Pharmacodynamic mismatch between the two components, one drug having additive /antagonistic effect leading to reduced efficacy or enhanced toxicity.

b. Pharmacokinetic mismatch and having peak efficacy at different time.

c. Chemical incompatibility leading to decreased therapeutic effect.

d. Interactions leading to reduced shelf life.

e. Drug interactions because of the common metabolizing pathways.

f. Limitations of finer dosing titration of individual ingredients.

Till recently, some the Indian firms were manufacturing the FDCs without the approval of Drugs Control General of India or the Central Drugs Standard Control Organization (CDSCO). Many states issued licenses to the firms for manufacture of FDCs without conducting any studies or counterchecks. In a detailed study on FDCs in three countries namely India, UK and USA during 2011-2012, it was found that 124 NSAID formulations (FDC) were available in India out of which only 34 were approved in India (27\%). During the period there were only six such FDCs in UK and 10 in USA. There were 25 Metformin FDC in India of which 20 were approve in India (80\%). Against the 25 Metformin FDCs in India there were only 8 in UK and 10 in USA and all were approved. Out of the 16 antidepressant/ benzodiazepine FDC formulations in India only three (19\%) were approved in India. There was only one approved antidepressant/ benzodiazepine FDC in USA and none were approved in UK. Though there were 10 anti-psychotic FDC formulations in India, only 3 were approved in India (30\%). There was only one anti-psychotic FDC in USA and none in UK. It is important to note that all the formulations of all categories available in UK and USA during the period in the respective therapeutic areas were approved by respective regulators. ${ }^{5}$

It was also found that 124 NSAID combinations gave rise to 2739 brands/products, 25 Metformin combinations to 536 products, 16 antidepressant/ benzodiazepine combinations to 301 products and 10 anti-psychotic formulations to 211 products. The unapproved FDCs gave rise to substantial proportion of branded products in the country. The sales of FDCs were almost $50 \%$ of total sales of NSAIDS and antidiabetic medicines sold in the country during the period 2011-2012. In the case of anti-psychotics it was one third and one fifth in the case of anti-depressants/benzodiazepines (Table 1). It is interesting to note that about, $81 \%$ of the marketed antidepressant/ benzodiazepine FDCs and $70 \%$ of the antipsychotic formulations were unapproved in India during the study period. Proportions of sales volumes arising from unapproved formulations were $69 \%$ from 
antidepressants/benzodiazepines and 43\% from antipsychotics; way ahead of parallel figures of $28 \%$ for NSAIDs and $0.4 \%$ for Metformincontaining unapproved FDC formulations. Over $53 \%$ of antipsychotic FDC sales came from formulations including drugs banned/restricted internationally. ${ }^{5}$

Table I Number of brands for certain groups of FDCs 20II-20I2

\begin{tabular}{llll}
\hline SI No & Therapeutic group & No of FDC & No of brands \\
\hline 1 & NSAID & 124 & 2739 \\
2 & Metformin combinations & 25 & 536 \\
3 & $\begin{array}{l}\text { Antidepressant/ } \\
\text { Benzodiazepine }\end{array}$ & 16 & 301 \\
4 & Anti-psychotics & 10 & 211 \\
\hline
\end{tabular}

Standing parliamentary committee report and banning of FDCs in 2016

The $59^{\text {th }}$ Standing Parliamentary Committee that investigated the functioning of Central Drugs Standard Control Organization (CDSCO) in 2012 observed that the CDSCO "had skewed priorities, according primacy to the propagation and facilitation of the drugs industry, due to which, the interest of the biggest stakeholder, i.e. the consumer has never been ensured". ${ }^{3}$ The report highlighted CDSCO's inability to produce any information related to the regulatory process that led to the approval of Deanxit, a fixed-dose combination of flupenthixol and melitracen. Deanxit is prohibited for sale and use in Denmark, its country of origin. The report observed that the approval to import and market Deanxit was in clear violation of the existing laws. ${ }^{6}$ The Parliamentary Committee pointed out that some State Licensing Authorities (SLAs) have been issuing manufacturing licenses for FDCs without prior clearance from Drugs Control General of India. About two thirds of the FDCs are manufactured and marketed in the country without the license of DCGI. Medicines banned or used under strict regulations were also found widely used as FDCs in India. Subsequently, the DCGI on $15^{\text {th }}$ January 2013 issued a circular directing the manufacturers to prove the safety and efficacy of FDCs licensed by the SLAs prior to $1^{\text {st }}$ October 2012, within 18 months. Those who fail to do so will be prohibited from manufacture and sales in India. However, FDCs approved before 1988 were exempted. In response to the circular, 6220 applications were submitted to the DCGI. ${ }^{7}$

On $16^{\text {th }}$ September 2014, Ministry of Health and Family Welfare constituted a Committee under the Chairmanship of Dr. CK Kokate, an academic expert of Pharmaceutical Sciences and former President of Pharmacy Council of India for examining the applications for rationality, safety, and efficacy of the FDCs. The committee submitted its final report to the Government of India on $10^{\text {th }}$ February 2016. The Government of India banned 344 FDCs under Section 26A of the Drugs and Cosmetics Act 1940 on $10^{\text {th }}$ March 2016. Out of them over 60 are combinations of antibiotics. The ban of 344 combinations affected about 7000 brands of a number of firms including major manufacturers like Abbott Healthcare, Macleods Pharma, Pfizer, Mankind, Alkem, IPCA, Medly, Glenmark, Franco Indian, Woekhardt and Aristo. The ban has affected annual sales to the tune of Rs 500 crores to 100 crores to certain firms. They have filed petitions in high courts at places like Delhi, Chennai and Bangalore against the ban of FDCs. In December 2016, the Delhi High Court stayed the ban of 344 FDCs imposed in March 2016 by the Government of India. The
Central Government approached the Supreme Court in January 2017 against the judgment of the Delhi High Court and the Supreme Court directed all High Courts in India to stop the hearing of all cases against the ban of the 344 FDCs and forward the cases to Supreme Court. The Supreme Court has directed the Government of India to classify the existing FDCs into four categories and submit to the Court, which is in progress Figure 1.

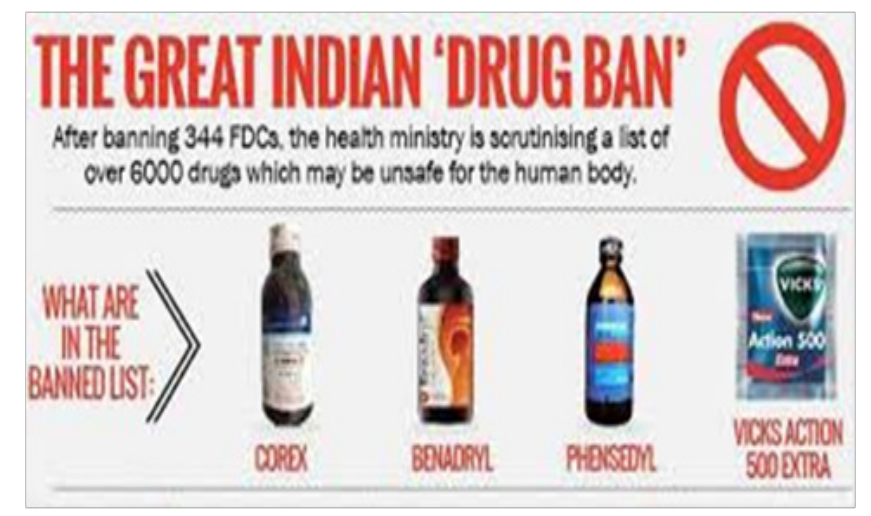

Figure I Some of the lead brands affected by Indian ban of 344 FDCs in 2016.

The new Pharmaceutical Policy 2017 of Government of India prescribes Bio-availability and Bio-equivalence tests (BA/BE Tests) in order ensure quality of medicines manufactured and marketed in the country. It should be made compulsory even for the future renewals of manufacturing licenses for all drugs. The Central Drug Regulator shall conduct regular annual audit of the laboratories which are accredited to conduct the $\mathrm{BA} / \mathrm{BE}$ tests and certify the results thereof. A road map for BA/BE implementation will be prepared and implemented by Central Drug Regulator. Provisions for self certification for BA/ BE compliance by existing Licensees would also be introduced so that effective quality standards can be ensured without waiting till the time of future renewals. Besides the Central Drug Regulator shall also get all manufacturing units inspected at least once annually through an accredited network of third party inspectors/agencies (national/ international) empanelled by it. Self-certification of manufacturing units can also be considered as an effective mechanism till such time that Central Drug Regulator develops capacity for annual inspections (Table 2).

Innovation in pharmaceuticals will be encouraged along with generic drugs in generic (salt names). As giving brand names to generic drugs hampers real innovation and that shall be discouraged. Public procurement and dispensing of drugs will be of generic drugs in salt names. To facilitate this, the government will pursue the policy of sale of single ingredient drugs by their Pharmacopoeial name / salt name. To keep the identity of the manufacturer, the manufacturer would be allowed to stamp its name on the drug package. In the case of patented drugs and Fixed Dose Combination (FDCs) drugs the manufacturers will be permitted to use brand names, if they want. But in such cases, the principle of 'one company - one drug - one brand name - one price' would be implemented.

\section{How to make the FDCs rational, scientific and need based?}

The FDCs in the Indian market increased tremendously in the last two decades and up to 2014. The increasing number and volume of FDCs is a matter of serious health concern in India. The DCGI 
could ban certain FDCs during the last two decades. However many new FDCs allegedly to be irrational were 'approved' during the same period and some states issued licenses without consulting the national licensing authority, Drugs Control General of India. It is a long process to ban the FDCs once they are approved in India. It is quite often difficult to prove one as irrational in Indian conditions. The DCGI and Drugs Technical Advisory Board (DTAB) have to play important roles in the process. FDCs have advantages when there is an identifiable patient population for whom treatment with a particular combination of actives in a fixed ratio of doses has been shown to be safe and effective and when all of the actives contribute to the overall therapeutic effect. In addition there can be real clinical benefits in the form of increased efficacy and/or a reduced incidence of adverse effects, but such claims should be supported by evidence. Additional advantages of FDCs are potentially lower costs of manufacturing compared to the costs of producing separate products administered concurrently, simpler logistics of distribution, improved patient adherence and reduced development of resistance as in the case of antimicrobials. Importantly, as for any new medicine the risks and benefits should be defined and compared.

Table 2 Products and Firms most affected of 2016 ban of 344 FDCs in India

\begin{tabular}{lcll}
\hline Categories impacted in \$ crore & \multicolumn{2}{l}{ Big losers in \$ crore } & \\
\hline Respiratory & 1,308 & Abbott Healthcare & 485 \\
Anti Diabetic & 609 & Macleods Pharma & 370 \\
Pain/Ananlgesics & 556 & Pfizer & 368 \\
Anti-infectives & 519 & Mankind & 253 \\
Gastro Intestinal & 485 & Alkem & 161 \\
Derma & 279 & Ipca & 130 \\
Neuro/CNS & 49 & Medley & 116 \\
Gynaecological & 30 & Glenmark & 110 \\
Blood Related & 3 & Franco & 104 \\
Urology & 1 & Wockhardt & 102 \\
Cardiac & 1 & Aristo & 102 \\
\hline
\end{tabular}

There cannot be difference of opinion regarding the need to weed out irrational and unethical combinations from Indian market for the safety of patients. The manufacturers don't dare to stop the production and sales of any of such combinations voluntarily. All the combinations which are marketed by companies in India are once permitted by the drug authorities. We cannot blame the manufacturers alone for the present situation. Quite often the drug authorities, particularly at the state level show an endorsing view in giving approval for the production and marketing of such preparations. The drug authorities give license, manufacturers produce and market the items and the doctors prescribe. The pharmacists dispense and promote! The new combinations are 'new drugs' as per the Drugs and Cosmetics Act (Rule 122 (E)). As a new drug each FDC has to undergo clinical trials and safety studies to qualify for entering the market. Is it really practiced in the country? The answer is currently 'No'. Who should be authorized to decide whether a FDC is rational or not? Is the Drug Control Department equipped with enough expertise in deciding the rational/irrational status of these drugs? Can we have a Committee, like the Pharmacopoeial Committee, at the national level to study and take care of the FDCs? Is it not necessary to make the clinical trials and other clinical research activities mandatory for approving FDCs? We need an effective mechanism to assess and prove that the FDC is rational and genuinely necessary in the context of our nation and its health issues. Drugs and Cosmetics Act and the Rules there need a lot of re-thinking in this regard.

The hospital pharmacists and the community pharmacists have to maintain the stock of FDCs and dispense them along with necessary information as and when prescribed. The multitude of FDCs often makes it very difficult for the pharmacists to remember which brand contains which drugs and in what combinations and proportions. The practicing pharmacists as professionals have to carry out various pharmaceutical care activities like patient counseling and drug information. In India currently there is no mechanism to provide the pharmacists and other health professionals the scientific and authoritative information and supporting literature regarding the FDCs approved by the Drugs control general of India (DCGI).

\section{Some other suggestions to make the FDCs rational in India are}

a. Pharmaceutical companies shall be forced to uphold the ethical concepts while introducing a FDC product. They have to conduct detailed studies on such combinations.

b. Pharmacokinetic drug interaction study and investigation on the safety profile shall be an integral part of all studies on FDC product.

c. The marketing approval for the new FDC shall be given by the DCGI only after subjecting for Pharmacoeconomic and pharmacovigilance studies for a specified period of time.

d. All the new and existing FDC products shall be subjected to a closely monitored national level post-marketing studies (Phase IV) involving identified community and hospital pharmacies in the country.

e. Education is an effective way to make the health care professionals including doctors and pharmacists aware of the ill effects of FDCs in India.

f. All Pharmacies including hospital and community pharmacies in which pharmacy students undergo training have to be educated about various aspect of FDCs and RDU.

\section{Acknowledgements}

None.

\section{Conflict of interest}

The author declares no conflict of interest.

\section{References}

1. Combination therapy is global strategy for treatment of malaria. Drugs for Neglected Diseases Initiative (DNDI). London, UK; 2005.

2. Goswami N, Gandhi A, Patel P, et al. An evaluation of knowledge, attitude and practices about prescribing fixed dose combinations among resident doctors. Perspect Clin Res. 2013;4(2):130-135.

3. Roy N, Madhiwalla N, Pai SA. Drug promotional practices in Mumbai: A qualitative study. Indian J Med Ethics. 2007;4(2):57-61.

4. Prakash S. Irrational combinations. No consideration for patient safety. Indian Journal of Pharmacology. 2007;39(5):217. 
5. McGettigan P, Roderick P, Mahajan R, et al. Use of Fixed Dose Combination Drugs in India: Central Regulatory Approval and Sales of FDCs containing NSAIDS, Metformin or Psychotropic Drugs. PLoS Med. 2015;12(5):e1001826.

6. $59^{\text {th }}$ Report, on the Functioning of the Central Drugs Standard Control Organization (CDSCO). Department-Related Standing Committee on Health and Family Welfare. New Delhi, Parliament of India; 2012. p. $1-115$.
7. Gupta YK, Ramachandran SS. Fixed dose drug combinations: Issues and challenges in India. Indian J Pharmacol. 2016;48(4):347-349. 